\title{
Research Article \\ Effective Natural Supersymmetry from the Yukawa Deflected Mediations
}

\author{
Tai-ran Liang, ${ }^{1}$ Bin Zhu, ${ }^{2}$ Ran Ding, ${ }^{3}$ and Tianjun $\mathrm{Li}^{4,5,6}$ \\ ${ }^{1}$ School of Physics and Electronic Information, Inner Mongolia University for the Nationalities, Tongliao 028043, China \\ ${ }^{2}$ Department of Physics, Yantai University, Yantai 264005, China \\ ${ }^{3}$ Center for High-Energy Physics, Peking University, Beijing 100871, China \\ ${ }^{4}$ Key Laboratory of Theoretical Physics and Kavli Institute for Theoretical Physics China (KITPC), Institute of Theoretical Physics, \\ Chinese Academy of Sciences, Beijing 100190, China \\ ${ }^{5}$ School of Physical Sciences, University of Chinese Academy of Sciences, Beijing 100049, China \\ ${ }^{6}$ School of Physical Electronics, University of Electronic Science and Technology of China, Chengdu 610054, China
}

Correspondence should be addressed to Bin Zhu; zhubin@mail.nankai.edu.cn

Received 28 April 2017; Accepted 13 June 2017; Published 10 July 2017

Academic Editor: Ning Chen

Copyright (C) 2017 Tai-ran Liang et al. This is an open access article distributed under the Creative Commons Attribution License, which permits unrestricted use, distribution, and reproduction in any medium, provided the original work is properly cited. The publication of this article was funded by SCOAP $^{3}$.

The natural supersymmetry (SUSY) requires light $(\leq 1 \mathrm{TeV})$ stop quarks, light sbottom quark, and gluinos. The first generation of squarks can be effectively larger than several $\mathrm{TeV}$ which does not introduce any hierarchy problem in order to escape the constraints from LHC. In this paper we consider a Yukawa deflected mediation to realize the effective natural supersymmetry where the interactions between squarks and messengers are made natural under certain Froggatt-Nielsen $U(1)_{X}$ charges. The first generation squarks obtain large and positive contribution from the Yukawa deflected mediation. The corresponding phenomenology and sparticle spectra are discussed in detail.

\section{Introduction}

Gauge Mediated SUSY Breaking (GMSB) [1] is an elegant framework. In its minimal form, the SUSY breaking hidden sector can be communicated with visible sector only through usual gauge interaction. It can be realized by introducing spurion field $X$ with $\langle X\rangle=M+\theta^{2} F$ and messenger fields $\Phi$ and the corresponding superpotential is written as

$$
W=X \Phi \bar{\Phi} .
$$

Here spurion $X$ couples to the SUSY breaking sector and $\langle X\rangle$ parameterizes the SUSY breaking effects and $\Phi$ are charged under the Standard Model $(\mathrm{SM}) S U(3) \times S U(2) \times U(1)$ gauge group. Since the mass matrix of scalar messenger components is not supersymmetric, the SUSY breaking effects from hidden sector can be mediated to visible sectors via messenger loops. Compared with gravity mediated SUSY breaking, GMSB has two obvious advantages:

(i) Soft terms are fully calculable. Even in the case of strongly coupled hidden sector, the soft terms can be still expressed as simple correlation functions of hidden sector, namely, the scenario of General Gauge Mediation (GGM) [2].

(ii) It is inherently flavor-conserving since gauge interaction is flavor-blinded and thus is strongly motivated by the SUSY flavor problem.

However, the status of minimal GMSB has been challenged after the discovery of SM-like Higgs boson with a mass of $125 \mathrm{GeV}[3,4]$. In order to lift Higgs mass to such desirable range, it then implies that Higgs mass should receive significant enhancement either from radiative corrections via stop/top loops [5, 6] or from extra tree-level sources [7]. The 
first option can be achieved through extremely heavy and unmixed stops or through lighter stops with maximal mixing (large trilinear soft term of stops) [8-11], while in minimal GMSB, the vanishing trilinear soft term at the messenger scale makes maximal mixing be impossible. The second option requires the extension of Minimal Supersymmetric Standard Model (MSSM) and has been widely investigated [12-27]. In this paper, we consider the first option where large trilinear term is required to soften fine-tuning. In fact, if the messenger sector is allowed to couple with squark or Higgs, the problem is improved with trilinear soft terms generated by additional interactions. The interactions can be generally divided into two types, that is, Higgs mediation and squark mediation. However, Higgs mediation generates irreducible positive contribution $\delta m_{H_{u}}^{2} \sim A_{H_{u}}^{2}$ and leads to large finetuning, which is the so-called $A / m_{H_{u}}^{2}$ problem. The situation is quite different in squark mediation since it does not suffer from such problem and it allows better control to fine-tuning. One compromise is that squark mediation reintroduces dangerous flavor problem since there is no prior reason to specify the hierarchy and alignment of Yukawa matrix of squark. In this direction, Froggatt-Nielsen (FN) mechanism [28] is adopted as a canonical solution. Here we take the same strategy for squark mediation. In a previous study, [29] considered the type of sfermion-sfermion-messenger interaction with FN mechanism. In this work, we extend the model to include sfermion-messenger-messenger interaction and to examine its phenomenology systematically.

The rest of this paper is layout as follows. In Section 2, we present our notation and model contents. The realization of FN mechanism in Supersymmetric Standard Models (SSMs) and $S U(5)$ models are reviewed in Section 3. In Section 4, the FN mechanism is extended to constrain the possible interactions between squarks and messengers. We show that a unique interaction can be obtained with appropriate charge assignment. In Section 5, we explore the phenomenology of this model with an emphasis on spectra and fine-tuning issues. The last section is devoted to conclusion.

\section{Vector-Like Particles (Messengers) in the SSMs and $S U(5)$ Models}

First, we list our convention for SSMs. We denote the lefthanded quark doublets, right-handed up-type quarks, righthanded down-type quarks, left-handed lepton doublets, right-handed neutrinos, and right-handed charged leptons as $Q_{i}, U_{i}^{c}, D_{i}^{c}, L_{i}, N_{i}^{c}$, and $E_{i}^{c}$, respectively. Also, we denote one pair of Higgs doublets as $H_{u}$ and $H_{d}$, which give masses to the up-type quarks/neutrinos and the down-type quark/charged leptons, respectively.

In this paper, we consider the messenger particles as the vector-like particles whose quantum numbers are the same as those of the SM fermions and their Hermitian conjugates. As we know, the generic vector-like particles do not need to form complete SU(5) or SO(10) representations in Grand Unified Theories. In particular it does not need complete multiplets (GUTs) from the orbifold constructions [30-41], intersecting
D-brane model building on Type II orientifold [42-44], Mtheory on $S^{1} / Z_{2}$ with Calabi-Yau compactifications [45-47], and F-theory with $U(1)$ fluxes [48-57] (For details, see [58]). Therefore, we will consider two kinds of supersymmetric models: (1) the SSMs with vector-like particles whose $U(1)_{X}$ charges can be completely different; (2) the SU(5) models.

In the SSMs, we introduce the following vector-like particles whose quantum numbers under $S U(3)_{C} \times S U(2)_{L} \times$ $U(1)_{Y}$ are given explicitly as follows:

$$
\begin{aligned}
& X Q+X Q^{c}=\left(3,2, \frac{1}{6}\right)+\left(\overline{3}, 2,-\frac{1}{6}\right) \\
& X U+X U^{c}=\left(3,1, \frac{2}{3}\right)+\left(\overline{3}, 1,-\frac{2}{3}\right) \\
& X D+X D^{c}=\left(3,1,-\frac{1}{3}\right)+\left(\overline{3}, 1, \frac{1}{3}\right) \\
& X L+X L^{c}=\left(1,2, \frac{-1}{2}\right)+\left(1,2, \frac{1}{2}\right) \\
& X E+X E^{c}=(1,1,-1)+(1,1,1) .
\end{aligned}
$$

In the SU(5) models, we have three families of the SM fermions whose quantum numbers under $S U(5)$ are

$$
\begin{aligned}
& F_{i}=10, \\
& \bar{f}_{i}=\overline{5},
\end{aligned}
$$

where $i=1,2,3$ for three families. The SM particle assignments in $F_{i}$ and $\bar{f}_{i}$ are

$$
\begin{aligned}
& F_{i}=\left(Q_{i}, U_{i}^{c}, E_{i}^{c}\right), \\
& \bar{f}_{i}=\left(D_{i}^{c}, L_{i}\right) .
\end{aligned}
$$

To break the SU(5) gauge symmetry and electroweak gauge symmetry, we introduce the adjoint Higgs field and one pair of Higgs fields whose quantum numbers under $S U(5)$ are

$$
\begin{aligned}
\Phi & =24, \\
H & =5, \\
\bar{H} & =\overline{5},
\end{aligned}
$$

where $H$ and $\bar{H}$ contain the Higgs doublets $H_{u}$ and $H_{d}$, respectively.

We consider the vector-like particles that form complete $S U$ (5) multiplets. The quantum numbers for these additional vector-like particles under the $S U(5) \times U(1)_{X}$ gauge symmetry are

$$
\begin{aligned}
& X F=\mathbf{1 0}, \\
& \overline{X F}=\overline{\mathbf{1 0}}, \\
& X f=\mathbf{5}, \\
& \overline{X f}=\overline{\mathbf{5}} .
\end{aligned}
$$


The particle contents from the decompositions of $X F, \overline{X F}$, $X f$, and $\overline{X f}$ under the SM gauge symmetries are

$$
\begin{aligned}
& X F=\left(X Q, X U^{c}, X E^{c}\right), \\
& \overline{X F}=\left(X Q^{c}, X U, X E\right), \\
& X f=\left(X D, X H_{u}\right), \\
& \overline{X f}=\left(X D^{c}, X H_{d}\right) .
\end{aligned}
$$

Here we have introduced two pairs of $X f$ and $\overline{X f}$, and we denote them as $X f_{i}$ and $\overline{X f}$, with $i=1,2$.

In this paper, we consider the messenger parity. We further consider the messenger parity, for example, discrete $Z_{n}$ symmetry with $n \geq 2$. Under this $Z_{n}$ symmetry, the vectorlike particles $X \Phi$ and $X \Phi^{c}$ transform are as follows:

$$
\begin{gathered}
X \Phi \longrightarrow \omega X \Phi, \\
X \Phi^{c} \longrightarrow \omega^{n-1} X \Phi^{c},
\end{gathered}
$$

where $\omega^{n}=1$. Thus, the lightest messenger will be stable. If the reheating temperature is lower than the mass of the lightest messenger, there is no cosmological problem. This is indeed work in our models. Otherwise, we can break the messenger parity a little bit by turning on tiny VEVs for $X L$ and/or $X L^{c}$.

In the gauge mediation, it is very difficult to obtain the Higgs boson with mass around $125.5 \mathrm{GeV}$ due to the small top quark trilinear soft $A_{t}$ term unless the stop quarks are very heavy around $10 \mathrm{TeV}$. To generate the large top quark trilinear soft $A_{t}$ term, we introduce the superpotential term $X Q X U^{c} H_{u}[59,60]$. In addition, we consider high scale gauge mediation by choosing

$$
\begin{aligned}
\langle S\rangle & \sim 10^{14} \mathrm{GeV}, \\
F_{S} & \sim 10^{20} \mathrm{GeV} .
\end{aligned}
$$

The point is that we can increase the magnitude of top quark trilinear soft term via RGE running. Another point is that the couplings between the spurion and messengers can be very small because $F_{S} /\langle S\rangle^{2} \sim 10^{-8}$.

\section{Froggatt-Nielsen Mechanism via an Anomalous $U(1)_{X}$ Gauge Symmetry}

It is well known that the SM fermion masses and mixings can be explained elegantly via the FN mechanism, where an additional flavor dependent global $U(1)_{X}$ symmetry is introduced. To stabilize this mechanism against quantum gravity corrections, we consider an anomalous gauged $U(1)_{X}$ symmetry. In a weakly coupled heterotic string theory, there exists an anomalous $U(1)_{X}$ gauge symmetry where the corresponding anomalies are cancelled by the Green-Schwarz mechanism [61-63]. For simplicity, we will not consider the $U(1)_{X}$ anomaly cancellation here, which can be done in general by introducing extra vector-like particles as in [64-67].
To break the $U(1)_{X}$ gauge symmetry, we introduce a flavon field $A$ with $U(1)_{X}$ charge -1 . To preserve SUSY close to the string scale, $A$ can acquire a VEV so that the $U(1)_{X}$ D-flatness can be realized. It was shown $[64,65]$ that

$$
0.171 \leq \epsilon \equiv \frac{\langle A\rangle}{M_{\mathrm{Pl}}} \leq 0.221,
$$

where $M_{\mathrm{Pl}}$ is the reduced Planck scale. Interestingly, $\epsilon$ is about the size of the Cabibbo angle. Also, the $U(1)_{X}$ charges of the SM fermions and the Higgs fields $\phi$ are denoted as $Q_{\phi}^{X}$.

The SM fermion Yukawa coupling terms arising from the holomorphic superpotential at the string scale in the SSMs are given by

$$
\begin{aligned}
-\mathscr{L}= & y_{i j}^{U}\left(\frac{A}{M_{\mathrm{Pl}}}\right)^{X Y U_{i j}} Q_{i} U_{j}^{c} H_{u} \\
& +y_{i j}^{D}\left(\frac{A}{M_{\mathrm{Pl}}}\right)^{X Y D_{i j}} Q_{i} D_{j}^{c} H_{d} \\
& +y_{i j}^{E}\left(\frac{A}{M_{\mathrm{Pl}}}\right)^{X Y E_{i j}} L_{i} E_{j}^{c} H_{d} \\
& +y_{i j}^{N}\left(\frac{A}{M_{\mathrm{Pl}}}\right)^{X Y N_{i j}} L_{i} N_{j}^{c} H_{u},
\end{aligned}
$$

where $y_{i j}^{U}, y_{i j}^{D}, y_{i j}^{E}$, and $y_{i j}^{N}$ are order one Yukawa couplings, and $X Y U_{i j}, X Y D_{i j}, X Y E_{i j}$, and $X Y N_{i j}$ are nonnegative integers:

$$
\begin{aligned}
& X Y U_{i j}=Q_{Q_{i}}^{X}+Q_{U_{j}^{c}}^{X}+Q_{H_{u}}^{X}, \\
& X Y D_{i j}=Q_{Q_{i}}^{X}+Q_{D_{j}^{c}}^{X}+Q_{H_{d}}^{X}, \\
& X Y E_{i j}=Q_{L_{i}}^{X}+Q_{E_{j}^{c}}^{X}+Q_{H_{d}}^{X}, \\
& X Y N_{i j}=Q_{L_{i}}^{X}+Q_{N_{j}^{c}}^{X}+Q_{H_{u}}^{X} .
\end{aligned}
$$

Similarly, the SM fermion Yukawa coupling terms in the $S U(5)$ models are

$$
\begin{aligned}
-\mathscr{L}= & y_{i j}^{U}\left(\frac{A}{M_{\mathrm{Pl}}}\right)^{X Y U_{i j}} F_{i} F_{j} H \\
& +y_{i j}^{D E}\left(\frac{A}{M_{\mathrm{Pl}}}\right)^{X Y D E_{i j}} F_{i} \bar{f}_{j} \bar{H} \\
& +y_{i j}^{N}\left(\frac{A}{M_{\mathrm{Pl}}}\right)^{X Y N_{i j}} \bar{f}_{i} N_{j}^{c} H,
\end{aligned}
$$

where

$$
\begin{gathered}
X Y U_{i j}=Q_{F_{i}}^{X}+Q_{F_{j}}^{X}+Q_{H}^{X}, \\
X Y D E_{i j}=Q_{F_{i}}^{X}+Q_{\bar{f}_{j}}^{X}+Q_{\bar{H}}^{X}, \\
X Y N_{i j}=Q_{\bar{f}_{i}}^{X}+Q_{N_{j}^{c}}^{X}+Q_{H}^{X} .
\end{gathered}
$$


TABLE 1: The quark textures in the SSMs and SU(5) models.

\begin{tabular}{lcr}
\hline Yukawa & The SSMs & SU(5) models \\
\hline$Y^{U}$ & $\left(\begin{array}{ccc}\epsilon^{8} & \epsilon^{5} & \epsilon^{3} \\
\epsilon^{7} & \epsilon^{4} & \epsilon^{2} \\
\epsilon^{5} & \epsilon^{2} & \epsilon^{0}\end{array}\right)$ & $\left(\begin{array}{ccc}\epsilon^{6} & \epsilon^{5} & \epsilon^{3} \\
\epsilon^{5} & \epsilon^{4} & \epsilon^{2} \\
\epsilon^{3} & \epsilon^{2} & \epsilon^{0}\end{array}\right)$ \\
$Y^{D}$ & $\epsilon^{c}\left(\begin{array}{ccc}\epsilon^{4} & \epsilon^{3} & \epsilon^{3} \\
\epsilon^{3} & \epsilon^{2} & \epsilon^{2} \\
\epsilon^{1} & \epsilon^{0} & \epsilon^{0}\end{array}\right)$ & $\epsilon^{c}\left(\begin{array}{ccc}\epsilon^{4} & \epsilon^{3} & \epsilon^{3} \\
\epsilon^{3} & \epsilon^{2} & \epsilon^{2} \\
\epsilon^{1} & \epsilon^{0} & \epsilon^{0}\end{array}\right)$ \\
\hline
\end{tabular}

In addition, we shall employ the quark textures for the SSMs and $S U(5)$ models in Table 1, which can reproduce the SM quark Yukawa couplings and the CKM quark mixing matrix for $\epsilon \approx 0.2$ [64-66]. And the following lepton textures can reproduce the neutrino masses and PMNS neutrino mixing matrix:

$$
\begin{gathered}
Y^{E} \sim \epsilon^{c}\left(\begin{array}{ccc}
\epsilon^{4} & \epsilon^{3} & \epsilon^{1} \\
\epsilon^{3} & \epsilon^{2} & \epsilon^{0} \\
\epsilon^{3} & \epsilon^{2} & \epsilon^{0}
\end{array}\right), \\
M_{L L} \sim \frac{\left\langle H_{u}\right\rangle^{2}}{M_{s}} \epsilon^{-5}\left(\begin{array}{ccc}
\epsilon^{2} & \epsilon^{1} & \epsilon^{1} \\
\epsilon^{1} & \epsilon^{0} & \epsilon^{0} \\
\epsilon^{1} & \epsilon^{0} & \epsilon^{0}
\end{array}\right),
\end{gathered}
$$

where $c$ is either $0,1,2$, or 3 , and $\tan \beta \equiv\left\langle H_{u}\right\rangle /\left\langle H_{d}\right\rangle$ satisfies $\epsilon^{c} \sim \epsilon^{3} \tan \beta$. This neutrino texture requires some amount of fine-tuning as it generically predicts

$$
\begin{aligned}
& \sin \theta_{12} \sim \epsilon, \\
& \Delta m_{12}^{2} \sim \Delta m_{23}^{2} .
\end{aligned}
$$

Interestingly, with $\epsilon$ as large as 0.2 , the amount of finetuning needed is not that huge and this is shown in the computer simulations of [64-66] with random values for the coefficients.

To be concrete, we choose the $U(1)_{X}$ charges for the SM fermions and Higgs fields in the SSMs as follows:

$$
\begin{aligned}
Q_{Q_{i}}^{X} & =(3,2,0), \\
Q_{U_{i}^{c}}^{X} & =(5,2,0), \\
Q_{D_{i}^{c}}^{X} & =(c+1, c, c), \\
Q_{L_{i}}^{X} & =(c+1, c, c), \\
Q_{E_{i}^{c}}^{X} & =(3,2,0), \\
Q_{H_{u}}^{X} & =Q_{H_{d}}^{X}=0,
\end{aligned}
$$

with $Q_{\phi_{i}}^{X} \equiv\left(Q_{\phi_{1}}^{X}, Q_{\phi_{2}}^{X}, Q_{\phi_{3}}^{X}\right)$ for the SM fermions $\phi_{i}$.
Also, we take the following $U(1)_{X}$ charges for the SM fermions and Higgs fields in the $S U(5)$ models:

$$
\begin{aligned}
Q_{F_{i}}^{X} & =(3,2,0), \\
Q_{f_{i}}^{X} & =(c+1, c, c), \\
Q_{H}^{X} & =Q_{\bar{H}}^{X}=0 .
\end{aligned}
$$

\section{Squark Mediation versus Higgs Mediation}

Natural SUSY can be regarded as an effective SUSY scenario where only stop, gluino, and small $\mu$ term are required in the spectra. As a consequence, the fine-tuning remains a manageable level. One nice property of Natural SUSY is that the first two generations of squarks can be very heavy without introducing any fine-tuning, which also evade bounds of SUSY direct searches from LHC. In terms of squark mediation with squark-messenger-messenger interaction, squarks receive additional positive contribution; thus it is possible to construct Natural SUSY model.

The basic formulas to compute corresponding soft terms are given as

$$
\begin{aligned}
A_{a b} & =-\frac{1}{32 \pi^{2}} d_{a}^{i j} \Delta\left(\lambda_{a i j}^{*} \lambda_{b i j}\right) \Lambda \\
\delta m_{a b}^{2} & =\frac{1}{256 \pi^{4}}\left(\frac{1}{2} d_{a}^{c B} d_{B}^{d e} \lambda_{a c B}^{*} \lambda_{b c C} \lambda_{d e B} \lambda_{d e C}^{*}\right. \\
& +d_{a}^{c B} d_{c}^{d C} \lambda_{a c B}^{*} \lambda_{b e B} \lambda_{c d C} \lambda_{d e C}^{*} \\
& +d_{a}^{c B} d_{b}^{d C} \lambda_{a c B}^{*} \lambda_{c e B} \lambda_{d e C}^{*} \lambda_{b d C} \\
& -d_{a}^{c d} d_{c}^{f B} y_{a c d}^{*} y_{b d e} \lambda_{c f B} \lambda_{e f B}^{*} \\
& +\frac{1}{2} d_{a}^{c B} d_{c}^{e f} y_{c e f} y_{d e f}^{*} \lambda_{a c B}^{*} \lambda_{b d B} \\
& +\frac{1}{2} d_{a}^{c d} d_{c}^{e f} y_{a c d}^{*} y_{c e f} \lambda_{b d B} \lambda_{e f B}^{*} \\
& +\frac{1}{2} d_{a}^{c B} d_{B}^{e f} \lambda_{a c B}^{*} \lambda_{e f B} y_{b c d} y_{d e f}^{*} \\
& \left.-2 d_{a}^{c B} C_{r}^{a c B} g_{r}^{2} \lambda_{a c B}^{*} \lambda_{b c B}\right) \Lambda^{2}
\end{aligned}
$$

where $\Lambda=F / M$ and $C_{r}^{i j k}=c_{r}^{i}+c_{r}^{j}+c_{r}^{k}$ is the sum of the quadratic Casimir of each field interacting through $\lambda_{i j k}$. In above expressions, we do not include the contributions from usual GMSB (thus is labeled by $\delta m_{a b}^{2}$ ) and all of indices are summed over except for $a$ and $b$. Without the FN mechanism, there will be general interaction between $Q_{i}, U_{i}$, and $D_{i}$. The squark mediation is not automatically minimal flavor violation like Higgs mediation. The MSSM-MSSM mixing term gives rise to dangerous nonvanishing and nondiagonal soft masses; for example,

$$
m_{Q_{1} Q_{2}}^{2} \sim \lambda_{q 1}^{2} \lambda_{q 2}^{2} \Lambda^{2}
$$

The nondiagonal terms in (20) motive [68] to construct chiral flavor violation scenario where only single $Q_{i}, U_{i}$, or $D_{i}$ is 
TABLE 2: Complete list of messenger fields and their $U(1)$ charge assignment.

\begin{tabular}{lcccccc}
\hline Messenger & $\left(X Q, X Q^{c}\right)$ & $\left(X U, X U^{c}\right)$ & $\left(X L, X L^{c}\right)$ & $\left(X D, X D^{c}\right)$ & $\left(X E, X E^{c}\right)$ & $X S$ \\
\hline$U(1)$ Charge & $(3,-3)$ & $(-5,5)$ & $(2,-2)$ & $(3,-3)$ & $(0,0)$ & 0 \\
\hline
\end{tabular}

allowed to couple the messenger. As a result, the dangerous flavor violation term is suppressed naturally. However our situation does not belong to chiral flavor violation. In order to realize effective SUSY scenario, all the first and second generation squarks must be coupled to messengers in order to obtain large soft masses enhancement. It seems the nondiagonal term is inevitable in (20). The loop hole comes from the fact that the bound is greatly improved when the squarks are nondegenerate. In particular, the largest bound comes from the first generation squarks because of large PDF effect of first generation quarks. Therefore it strongly suggests us for considering the first generation squark mediation which is technically natural under FN mechanism. The FN natural model is free from MSSM-MSSM mixing and the formulas are reduced to

$$
\begin{aligned}
A_{a} & =-\frac{1}{16 \pi^{2}} d_{a}^{c B} \lambda_{a c B}^{2} \Lambda, \\
\delta m_{a}^{2} & =\frac{1}{256 \pi^{4}}\left(\frac{1}{2} d_{a}^{c B} d_{B}^{d e}\left|\lambda_{a c B}\right|^{2}\left|\lambda_{d e B}\right|^{2}\right. \\
& +d_{a}^{c B} d_{c}^{d C}\left|\lambda_{a c B}\right|^{2}\left|\lambda_{c d C}\right|^{2}+d_{a}^{c B} d_{a}^{d C}\left|\lambda_{a c B}\right|^{2}\left|\lambda_{a d C}\right|^{2} \\
& -d_{a}^{c d} d_{c}^{f B}\left|y_{a c d}\right|^{2}\left|\lambda_{c f B}\right|^{2}+\frac{1}{2} d_{a}^{c B} d_{c}^{e f}\left|y_{c e f}\right|^{2}\left|\lambda_{a c B}\right|^{2} \\
& +\frac{1}{2} d_{a}^{c d} d_{c}^{e f} y_{a c d}^{*} y_{c e f} \lambda_{a d B} \lambda_{e f B}^{*} \\
& \left.+\frac{1}{2} d_{a}^{c B} d_{B}^{e f} \lambda_{a c B}^{*} \lambda_{e f B} y_{a c d} y_{d e f}^{*}-2 d_{a}^{c B} C_{r}^{a c B} g_{r}^{2}\left|\lambda_{a c B}\right|^{2}\right) \\
& \cdot \Lambda^{2} .
\end{aligned}
$$

It is easy to demonstrate how FN mechanism makes the squark mediation flavor-blinded. The general squarkmessenger-messenger interaction within the messenger sector being $S U(5)$ complete multiplets is divided into Q-type, $U$-type, and $D$-type mediations; here $U$ and $D$ denote $\bar{u}$ and $\bar{d}$, respectively. In Table 2, we list the complete messenger fields and their $U(1)$ charge assignment. tial is

For the Q-type Mediation, the most general superpoten-

$$
\begin{aligned}
W_{\mathrm{Q}}= & \lambda_{q 1_{i}} Q_{i} X Q^{c} X S+\lambda_{q 2_{i}} Q_{i} X D^{c} X L \\
& +\lambda_{q 3_{i}} Q_{i} X U^{c} X L^{c}+\lambda_{q 4_{i}} X Q X D,
\end{aligned}
$$

where $i=1, \ldots, 3$ is family indices. Based on Table 2, the Yukawa couplings in Q-type mediation can be determined as follows:

$$
\begin{aligned}
& \lambda_{q 1_{i}} \sim\left\{1, \frac{1}{\epsilon}, \frac{1}{\epsilon^{3}}\right\}, \\
& \lambda_{q 2_{i}} \sim\left\{\epsilon^{2}, \epsilon, \frac{1}{\epsilon}\right\},
\end{aligned}
$$

$$
\begin{aligned}
& \lambda_{q 3_{i}} \sim\left\{\epsilon^{6}, \epsilon^{5}, \epsilon^{3}\right\}, \\
& \lambda_{q 4_{i}} \sim\left\{\epsilon^{9}, \epsilon^{8}, \epsilon^{6}\right\} .
\end{aligned}
$$

Terms with negative power of $\epsilon$ must be removed in order not to violate the holomorphic requirement of superpotential. While terms with positive order of $\epsilon$ can be ignored which is guaranteed by the smallness of $\epsilon$. Therefore only $\lambda_{q 1}$ is allowed under the consideration of FN mechanism and holomorphy. For now we only consider squark-messenger-messenger interaction; this is mainly because the squark-squarkmessenger interaction under FN charges has been discussed in the literature [69]. Since only the $Q_{1}$ mediation is allowed, there is no flavor-changing problem.

For the $U$-type mediation the most general superpotential is

$$
\begin{aligned}
W_{U}= & \lambda_{u 1_{i}} U_{i} X U X S+\lambda_{u 2_{i}} U_{i} X D^{c} X D^{c} \\
& +\lambda_{u 3_{i}} U X Q X L^{c}+\lambda_{u 4_{i}} U_{i} X E X D .
\end{aligned}
$$

According to FN mechanism the coupling looks like

$$
\begin{aligned}
& \lambda_{u 1_{i}} \sim\left\{1, \frac{1}{\epsilon^{3}}, \frac{1}{\epsilon^{5}}\right\}, \\
& \lambda_{u 2_{i}} \sim\left\{\frac{1}{\epsilon}, \frac{1}{\epsilon^{4}}, \frac{1}{\epsilon^{6}}\right\}, \\
& \lambda_{u 3_{i}} \sim\left\{\epsilon^{6}, \epsilon^{3}, \epsilon\right\}, \\
& \lambda_{u 4_{i}} \sim\left\{\epsilon^{2}, \frac{1}{\epsilon}, \frac{1}{\epsilon^{3}}\right\} .
\end{aligned}
$$

It is similar to $Q$-type mediation; only $\lambda_{u 1_{1}}$ is allowed. For $D$ type mediation we have

$$
\begin{aligned}
W_{D}= & \lambda_{d 1_{i}} D_{i} X Q X L^{c}+\lambda_{d 2_{i}} D_{i} X Q^{c} X Q^{c} \\
& +\lambda_{d 3_{i}} D_{i} X D^{c} X U^{c}+\lambda_{d 4_{i}} D_{i} X E^{c} X U .
\end{aligned}
$$

Subject to the FN mechanism we obtain the couplings

$$
\begin{aligned}
& \lambda_{d 1_{i}} \sim\left\{\epsilon^{6}, \epsilon^{5}, \epsilon^{5}\right\}, \\
& \lambda_{d 2_{i}} \sim\left\{\frac{1}{\epsilon}, \frac{1}{\epsilon^{2}}, \frac{1}{\epsilon^{2}}\right\}, \\
& \lambda_{d 3_{i}} \sim\left\{\epsilon^{7}, \epsilon^{6}, \epsilon^{6}\right\}, \\
& \lambda_{d 4_{i}} \sim\left\{1, \frac{1}{\epsilon}, \frac{1}{\epsilon}\right\} .
\end{aligned}
$$

Consequently the allowed Yukawa deflected mediation interaction for squarks is summarized as follows:

$$
W=\lambda_{q} Q_{1} X Q^{c} X S+\lambda_{u} U_{i} X U X S+\lambda_{d} D_{i} X E^{c} X U .
$$


From (28), we obtain the extra contribution to soft masses for the first generation squarks. In other words there is no desirable large trilinear term $A_{t}$ from (28) which motivates us to resort to Higgs mediation.

Based on FN mechanism the only allowed superpotential for Higgs mediation is

$$
W_{H}=\lambda_{h} H_{u} X D^{c} X Q \text {. }
$$

It automatically preserves minimal flavor violation (MFV). Using (21), we obtain following soft terms:

$$
\begin{aligned}
& A_{t}=-\frac{3 \Lambda \lambda_{h}^{2}}{16 \pi^{2}} \\
& \delta m_{H_{u}}^{2} \\
& \quad=\frac{\Lambda^{2}\left(18 \lambda_{h}^{4}-6\left(7 g_{1}^{2} / 30+3 g_{2}^{2} / 2+8 g_{3}^{2} / 3\right) \lambda_{h}^{2}\right)}{256 \pi^{4}}, \\
& \delta m_{Q_{3}}^{2}=-\frac{3 \Lambda^{2} \lambda_{h}^{2} y_{t}^{2}}{256 \pi^{4}}, \\
& \delta m_{U_{3}}^{2}=-\frac{3 \Lambda^{2} \lambda_{h}^{2} y_{t}^{2}}{128 \pi^{4}}, \\
& \delta m_{\mathrm{Q}_{1}}^{2}=\frac{\Lambda^{2}\left(8 \lambda_{q}^{4}-2\left(g_{1}^{2} / 30+3 g_{2}^{2} / 2+8 g_{3}^{2} / 3\right) \lambda_{q}^{2}\right)}{256 \pi^{4}}, \\
& \delta m_{U_{1}}^{2} \\
& =\frac{\Lambda^{2}\left(5 \lambda_{u}^{4}-2\left(13 g_{1}^{2} / 30+3 g_{2}^{2} / 2+8 g_{3}^{2} / 3\right) \lambda_{u}^{2}\right)}{256 \pi^{4}}, \\
& \delta m_{D_{1}}^{2}=\frac{\Lambda^{2}\left(5 \lambda_{d}^{4}-2\left(14 g_{1}^{2} / 15+8 g_{3}^{2} / 3\right) \lambda_{d}^{2}\right)}{256 \pi^{4}} .
\end{aligned}
$$

The choice of Higgs mediation in (29) is crucial in reducing the fine-tuning:

(i) The trilinear soft term has an overall factor 3 coming from the higher representation of $S U(5)$. Thus it can give rise to large trilinear term compared with other Higgs mediation.

(ii) $m_{H_{u}}^{2}$ has a negative contribution from $S U(3)$ gauge coupling. Such a large coupling can reduce the finetuning easily.

The parameter space is thus determined by the following parameters:

$$
\left\{\Lambda, M, \lambda_{q}, \lambda_{u}, \lambda_{d}, \lambda_{h}, \tan \beta, \operatorname{sign}(\mu)\right\} .
$$

\section{Phenomenology Analysis}

In this section, we give a detailed discussion on the results from our effective supersymmetry model. In particular the Higgs mass, stop mass, gluino mass, and fine-tuning are given explicitly. In our numerical analysis, the relevant soft terms are firstly generated at messenger scale in terms of gauge mediation and Higgs and squark mediation. The low scale soft terms are obtained by solving the two-loop RG equations. For this purpose, we implemented the corresponding boundary conditions in (30) into the SARAH [70-74] package. Then SARAH is used to create a SPheno $[75,76]$ version for the MSSM to calculate particle spectrum. The tasks of parameter scans are implemented using package SSP [77].

The framework that we concentrate on is MSSM with Yukawa deflected mediation. Its input parameters are given in (31). The scan range we adapt is

$$
\begin{aligned}
& \Lambda \in\left(6 \times 10^{4}, 6 \times 10^{5}\right) \mathrm{GeV}, \\
& \lambda_{h} \in(0,1.2) .
\end{aligned}
$$

Other parameters are fixed to $M=10^{8} \mathrm{GeV}, \tan \beta=10$, and $\operatorname{sign}(\mu)=1$. For the parameters in squark mediation, we divide it into two scenarios:

Degenerated squark: $\lambda_{q}=\lambda_{u}=\lambda_{d}=0$,

Nondegenerated squark: $\lambda_{q}=\lambda_{u}=\lambda_{d}=1.2$.

In the scan, various mass spectrum and low energy constraints have been considered and listed as follows:

(1) The Higgs mass constraints:

$$
123 \mathrm{GeV} \leq m_{h} \leq 127 \mathrm{GeV} .
$$

(2) LEP bounds and $B$ physics constraints:

$$
\begin{aligned}
& 1.6 \times 10^{-9} \leq B R\left(B_{s} \rightarrow \mu^{+} \mu^{-}\right) \leq 4.2 \times 10^{-9}(2 \sigma) \\
& {[78],} \\
& 2.99 \times 10^{-4} \leq B R(b \rightarrow s \gamma) \leq 3.87 \times 10^{-4}(2 \sigma) \\
& {[79],} \\
& 7.0 \times 10^{-5} \leq B R\left(B_{u} \rightarrow \tau \nu_{\tau}\right) \leq 1.5 \times 10^{-4}(2 \sigma) \\
& {[79] .}
\end{aligned}
$$

(3) Sparticle bounds from LHC Run-II:

(i) Light stop mass $m_{\tilde{t}_{1}}>850 \mathrm{GeV}[80,81]$,

(ii) Light sbottom mass $m_{\tilde{b}_{1}}>840-1000 \mathrm{GeV}[82$, 83],

(ii) Degenerated first two generation squarks (both left-handed and right-handed) $m_{\tilde{q}}>1000-$ $1400 \mathrm{GeV}$ [83],

(iv) Gluino mass $m_{\tilde{g}}>1800 \mathrm{GeV}[81,84]$.

Finally, Barbieri-Giudice measure $[85,86]$ is used to quantify the fine-tuning:

$$
\Delta_{\mathrm{FT}} \equiv \max \left\{\Delta_{a}\right\}, \quad \text { where } \Delta_{a} \equiv \frac{\partial \log m_{Z}^{2}}{\partial \log a},
$$

where $a$ denotes the input parameters in (31). 


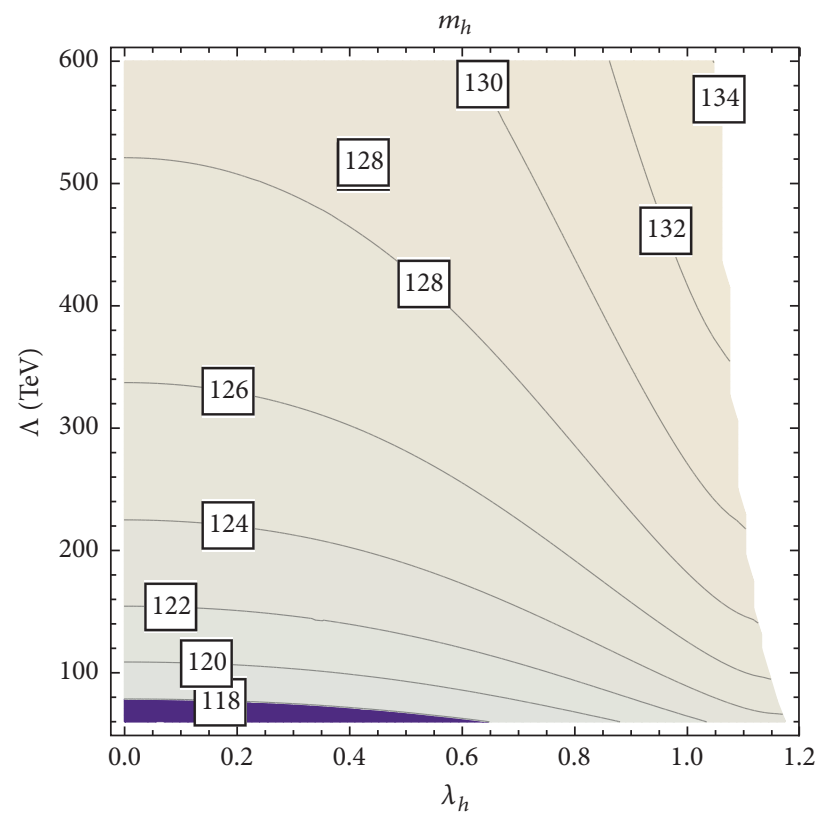

FIgURE 1: Distribution of Higgs mass in $\left[\lambda_{h}, \Lambda\right]$ plane.

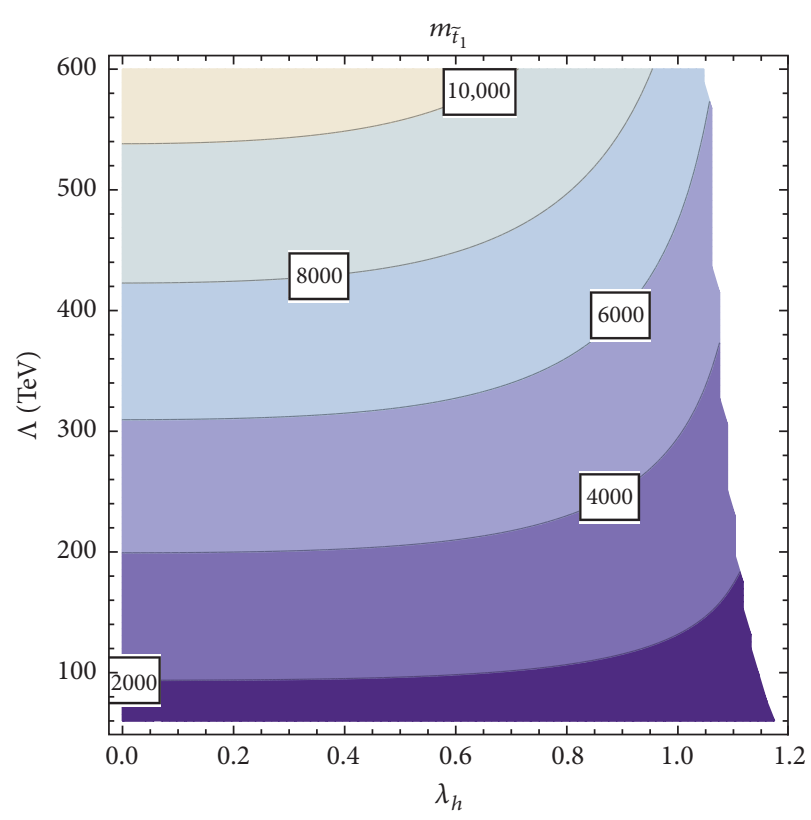

(a)

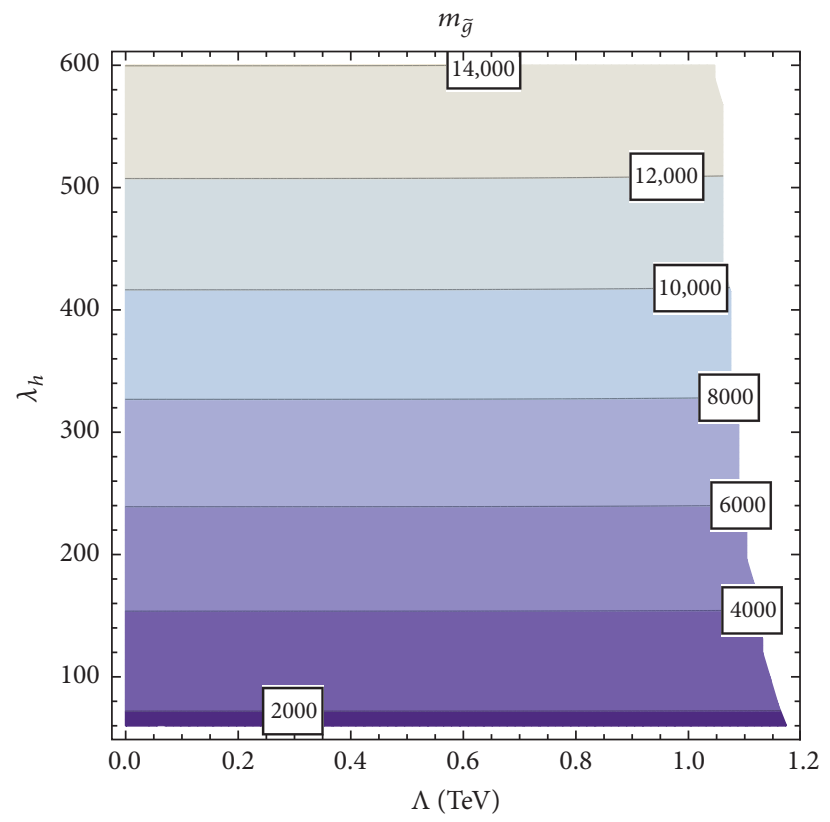

(b)

Figure 2: Distributions of stop (a) and gluino mass (b) in $\left[\lambda_{h}, \Lambda\right]$ plane.

In Figures 1-3, we display the contour plots of important mass spectra and fine-tuning measure $\Delta_{\mathrm{FT}}$ in the $\left[\lambda_{h}, \Lambda\right]$ plane. There are some notable features which can be learned from these figures and summarized as follows:

(1) The Higgs Mass. The Higgs mass range is taken from $123 \mathrm{GeV}$ to $127 \mathrm{GeV}$ in our numerical analysis. For small $\lambda_{h}$, one expects Higgs mass simply growth with an increases of $\Lambda$. When $\lambda_{h}$ increases, the allowed parameter space is forced to shift to smaller $\Lambda$ region in order to obtain correct Higgs mass.

(2) The Fine-Tuning Measure. For small values of $\Lambda$ and $\lambda_{h}, \Delta_{\mathrm{FT}}$ is usually dominated by $\Lambda$. Since in these regions the RGE effects are most important, the contribution to the fine-tuning of $\lambda_{h}$, which only 


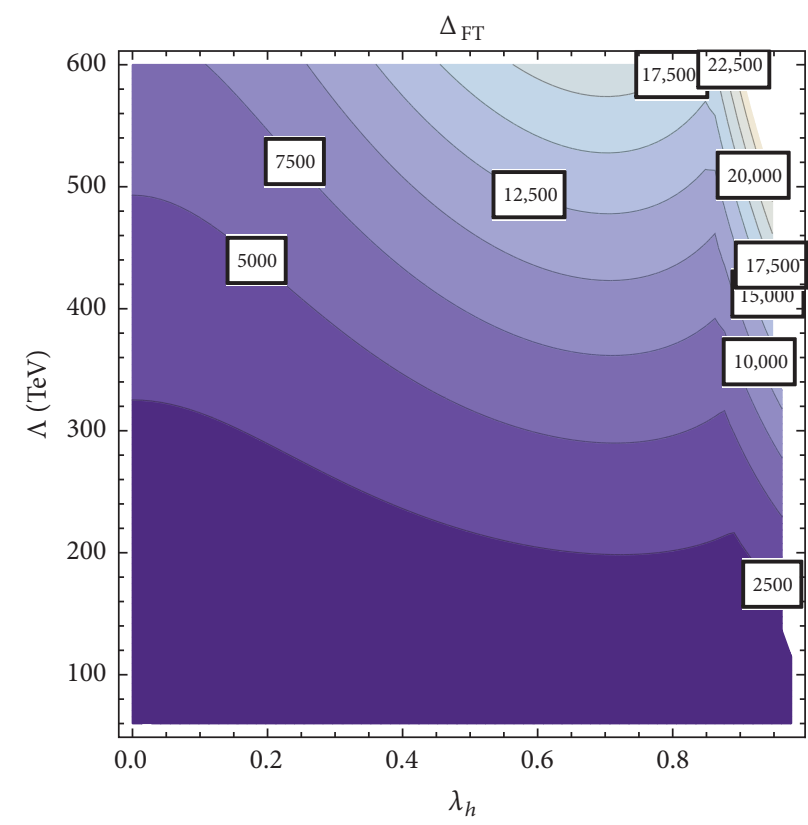

FIGURE 3: Distribution of fine-tuning measure in $\left[\lambda_{h}, \Lambda\right]$ plane.

affects the boundary conditions, is negligible. The important parameter thus is $\Lambda$ which sets the range of the RGE running. For moderate $\Lambda_{a}$ and $\lambda_{h}$, the contributions from $\mu$ and $\Lambda$ are almost comparable. When $\lambda_{h}$ becomes large it is always the biggest contributor to fine-tuning measure independent of the value of $\Lambda$.

(3) The Squark and Gluino Masses. Both stop and gluino masses fall into multi-TeV range and therefore out of current LHC reach.

\section{Conclusions}

In this paper, we investigated the extended gauge mediation models where Yukawa interaction between messengers and matter superfields is made natural under the consideration of F-N U(1) symmetry. Because of Higgs mediation the large A-term is generated naturally which can be used to enhance the Higgs mass efficiently. Considering the additional quark mediation, it is found that first generation squarks get large positive contribution, thus escaping from dangerous LHC constraints. We further study the parameter space and phenomenology numerically. The results show that the model is still promising under the stringent LHC constraint.

\section{Conflicts of Interest}

The authors declare that they have no conflicts of interest.

\section{Acknowledgments}

This research was supported in part by the Natural Science Foundation of China under Grant nos. 10821504, 11075194, 11135003 , and 11275246 .

\section{References}

[1] G. F. Giudice and R. Rattazzi, “Theories with gauge-mediated supersymmetry breaking," Physics Reports, vol. 322, pp. 419499, 1999.

[2] P. Meade, N. Seiberg, and D. Shih, "General Gauge Mediation," Progress of Theoretical Physics Supplement, vol. 177, pp. 143-158, 2009.

[3] P. Draper, P. Meade, M. Reece, and D. Shih, "Implications of a $125 \mathrm{GeV}$ Higgs boson for the MSSM and low-scale supersymmetry breaking," Physical Review D, vol. 85, Article ID 095007, 2012.

[4] M. A. Ajaib, I. Gogoladze, F. Nasir, and Q. Shafi, "Revisiting mGMSB in light of a $125 \mathrm{GeV}$ Higgs," Physics Letters B, vol. 713, no. 4-5, pp. 462-468, 2012.

[5] S. Heinemeyer, O. Stal, and G. Weiglein, "Interpreting the LHC Higgs search results in the MSSM," Physics Letters B, vol. 710, no. 1, pp. 201-206, 2012.

[6] A. Arbey, M. Battaglia, A. Djouadi, F. Mahmoudi, and J. Quevillon, "Implications of a $125 \mathrm{GeV}$ Higgs for supersymmetric models," Physics Letters B, vol. 708, no. 1-2, pp. 162-169, 2012.

[7] L. J. Hall, D. Pinner, and J. T. Ruderman, "A natural SUSY Higgs near 125 GeV," Journal of High Energy Physics, vol. 131, 2012.

[8] J. Casas, J. Espinosa, M. Quirós, and A. Riotto, "Erratum: The lightest Higgs boson mass in the Minimal Supersymmetric Standard Model," Nuclear Physics B, vol. 439, no. 1-2, pp. 466468, 1995.

[9] M. Carena, J. R. Espinosa, M. Quirós, and C. E. M. Wagner, "Analytical expressions for radiatively corrected Higgs masses and couplings in the MSSM," Physics Letters B, vol. 355, pp. 209221, 1995.

[10] J. A. Evans and D. Shih, "Surveying extended GMSB models with $m_{h}=125$ GeV," Journal of High Energy Physics, vol. 93, 2013.

[11] H. E. Haber, R. Hempfling, and A. H. Hoang, "Approximating the radiatively corrected Higgs mass in the minimal supersymmetric model," Zeitschrift für Physik C Particles and Fields, vol. 75, no. 3, pp. 539-554, 1997.

[12] U. Ellwanger, "A Higgs boson near $125 \mathrm{GeV}$ with enhanced diphoton signal in the NMSSM," Journal of High Energy Physics, vol. 44, 2012.

[13] P. Fileviez Pérez, "SUSY spectrum and the Higgs mass in the BLMSSM," Physics Letters B, vol. 711, no. 5, pp. 353-359, 2012.

[14] F. Boudjema and G. Drieu La Rochelle, "Beyond the MSSM Higgs bosons at $125 \mathrm{GeV}$," Physical Review D, vol. 86, no. 1, Article ID 015018, 2012.

[15] K. S. Jeong, Y. Shoji, and M. Yamaguchi, "Singlet-doublet Higgs mixing and its implications on the Higgs mass in the PQNMSSM," Journal of High Energy Physics, vol. 7, 2012.

[16] H. An, T. Liu, and L. T. Wang, " $125 \mathrm{GeV}$ Higgs boson, enhanced diphoton rate, and gauged $U(1)_{\mathrm{PQ}}$-extended MSSM," Physical Review D, vol. 86, Article ID 075030, 2012.

[17] E. Hardy, J. March-Russell, and J. Unwin, "Precision unification in $\lambda$ SUSY with a $125 \mathrm{GeV}$ Higgs," Journal of High Energy Physics, vol. 72, 2012.

[18] M. Hirsch, W. Porod, L. Reichert, and F. Staub, "Phenomenology of a supersymmetric $U(1)_{B-L} \times U(1)_{R}$ extension of the standard model with inverse seesaw mechanism," Physical Review D, vol. 86, Article ID 093018, 2012.

[19] T. Basak and S. Mohanty, "Triplet-singlet extension of the MSSM with a $125 \mathrm{GeV}$ Higgs boson and dark matter," Physical Review D, vol. 86, Article ID 075031, 2012. 
[20] K. Schmidt-Hoberg and F. Staub, "Enhanced $h \rightarrow \gamma \gamma$ rate in MSSM singlet extensions," Journal of High Energy Physics, vol. 195, 2012.

[21] K. Benakli, M. D. Goodsell, and F. Staub, "Dirac gauginos and the 125 GeV Higgs," Journal of High Energy Physics, vol. 73, 2013.

[22] X. Lu, H. Murayama, J. T. Ruderman, and K. Tobioka, "Natural Higgs Mass in Supersymmetry from Nondecoupling Effects," Physical Review Letters, vol. 112, Article ID 191803, 2014.

[23] E. Bertuzzo, C. Frugiuele, T. Gregoire, and E. Ponton, "Dirac gauginos, R symmetry and the $125 \mathrm{GeV}$ Higgs," Journal of High Energy Physics, vol. 89, 2015.

[24] F. Staub, "Beyond-MSSM Higgs sectors," Proceeding of Science, vol. 2014, 2014.

[25] R. Ding, T. Li, F. Staub, C. Tian, and B. Zhu, "Supersymmetric standard models with a pseudo-Dirac gluino from hybrid $F$ and D-term supersymmetry breaking," Physical Review D, vol. 92, no. 1, Article ID 015008, 2015.

[26] C. Alvarado, A. Delgado, A. Martin, and B. Ostdiek, "Dirac triplet extension of the MSSM," Physical Review D, vol. 92, no. 3, Article ID 035009, 2015.

[27] P. Bandyopadhyay, C. Corianò, and A. Costantini, "Perspectives on a supersymmetric extension of the standard model with a $Y=0$ Higgs triplet and a singlet at the LHC," Journal of High Energy Physics, vol. 45, 2015.

[28] C. D. Froggatt and H. B. Nielsen, "Hierarchy of quark masses, cabibbo angles and CP violation," Nuclear Physics B, vol. 147, no. 3-4, pp. 277-298, 1979.

[29] Y. Shadmi and P. Z. Szabo, "Flavored gauge-mediation," Journal of High Energy Physics, vol. 124, 2012.

[30] Y. Kawamura, "Gauge symmetry reduction from the extra space $S^{1} / Z_{2}$," Progress of Theoretical Physics, vol. 103, no. 3, pp. 613-619, 2000 .

[31] Y. Kawamura, "Triplet-doublet splitting, proton stability and an extra dimension," Progress of Theoretical Physics, vol. 105, no. 6, pp. 999-1006, 2001.

[32] Y. Kawamura, "Split multiplets, coupling unification and an extra dimension," Progress of Theoretical Physics, vol. 105, pp. 691-696, 2001.

[33] G. Altarelli and F. Feruglio, " $S U(5)$ grand unification in extra dimensions and proton decay," Physics Letters B, vol. 511, no. 2-4, pp. 257-264, 2001.

[34] L. Hall and Y. Nomura, "Gauge unification in higher dimensions," Physical Review D, vol. 64, no. 5, Article ID 055003, 2001.

[35] A. Hebecker and J. March-Russell, "A minimal $S^{1} /\left(Z_{2} \times Z_{2}^{\prime}\right)$ orbifold GUT," Nuclear Physics B, vol. 613, no. 1-2, pp. 3-16, 2001.

[36] T. Li, "GUT breaking on $M^{4} \times T^{2} /\left(Z_{2} \times Z_{2}^{\prime}\right)$," Physics Letters $B$, vol. 520, no. 3-4, pp. 377-384, 2001.

[37] T. Li, " $N=2$ supersymmetric GUT breaking on $T^{2}$ orbifolds," Nuclear Physics B, vol. 619, no. 1-3, pp. 75-104, 2001.

[38] R. Dermíšek and A. Mafi, "SO(10) grand unification in five dimensions: proton decay and the $\mu$ problem," Physical Review $D$, vol. 65, no. 5, Article ID 055002, 2002.

[39] T. Li, "Gauge symmetry and supersymmetry breaking by discrete symmetry," Nuclear Physics B, vol. 633, no. 1-2, pp. 83-96, 2002.

[40] I. Gogoladze, Y. Mimura, and S. Nandi, "Unification of gauge, Higgs and matter in extra dimensions," Physics Letters B, vol. 562, no. 3-4, pp. 307-315, 2003.

[41] I. Gogoladze, Y. Mimura, and S. Nandi, "Unity of elementary particles and forces in higher dimensions," Physical Review Letters, vol. 91, no. 14, Article ID 141801, 2003.
[42] R. Blumenhagen, M. Cvetič, P. Langacker, and G. Shiu, "Toward realistic intersecting D-brane models," Annual Review of Nuclear and Particle Science, vol. 55, pp. 71-139, 2005.

[43] M. Cvetič, I. Papadimitriou, and G. Shiu, "Erratum to: "Supersymmetric three family $S U(5)$ grand unified models from type IIA orientifolds with intersecting D6-branes"," Nuclear Physics $B$, vol. 696, no. 1-2, p. 298, 2004.

[44] C. M. Chen, T. Li, and D. V. Nanopoulos, "Flipped and unflipped $S U(5)$ as type IIA flux vacua," Nuclear Physics B, vol. 751, no. 1-2, pp. 260-284, 2006.

[45] V. Braun, Y.-H. He, B. A. Ovrut, and T. Pantev, "A heterotic standard model," Physics Letters B, vol. 618, no. 1-4, pp. 252-258, 2005.

[46] V. Braun, Y. H. He, B. A. Ovrut, and T. Pantev, "The exact MSSM spectrum from string theory," Journal of High Energy Physics, vol. 2006 , no. $5,2006$.

[47] V. Bouchard and R. Donagi, "An SU(5) heterotic standard model," Physics Letters B, vol. 633, no. 6, pp. 783-791, 2006.

[48] C. Vafa, "Evidence for F-theory," Nuclear Physics B, vol. 469, no. 3, pp. 403-415, 1996.

[49] R. Donagi and M. Wijnholt, "Model Building with F-Theory," Advances in Theoretical and Mathematical Physics, vol. 15, no. 5, pp. 1237-1317, 2011.

[50] C. Beasley, J. J. Heckman, and C. Vafa, "GUTs and exceptional branes in F-theory - I," Journal of High Energy Physics, vol. 2009, no. 1, p. 58, 2009.

[51] C. Beasley, J. J. Heckman, and C. Vafa, "GUTs and exceptional branes in F-theory-II. Experimental predictions," Journal of High Energy Physics, vol. 2009, no. 1, p. 59, 2009.

[52] R. Donagi and M. Wijnholt, "Breaking GUT groups in Ftheory," Advances in Theoretical and Mathematical Physics, vol. 15, no. 6, pp. 1523-1603, 2011.

[53] A. Font and L. E. Ibáñez, "Yukawa structure from $U(1)$ fluxes in F-theory grand unification," Journal of High Energy Physics, vol. 2009, no. 2, 2009.

[54] J. Jiang, T. Li, D. V. Nanopoulos, and D. Xie, “F-SU(5),” Physics Letters B, vol. 677, no. 5325, p. 322, 2009.

[55] R. Blumenhagen, "Gauge coupling unification in F-theory grand unified theories," Physical Review Letters, vol. 102, no. 7, Article ID 071601, 2009.

[56] J. Jiang, T. Li, D. V. Nanopoulos, and D. Xie, "Flipped $S U(5) \times$ $U(1)_{X}$ models from F-theory," Nuclear Physics B, vol. 830, no. 1, pp. 195-220, 2010.

[57] T. Li, "SU(5) and $S O(10)$ Models from F-Theory with Natural Yukawa Couplings," Physical Review D, vol. 81, no. 6, Article ID 065018, 2010.

[58] T. Li and D. V. Nanopoulos, "General gauge and anomaly mediated supersymmetry breaking in grand unified theories with vector-like particles," Journal of High Energy Physics, vol. 90, pp. 1-39, 2011.

[59] Z. Kang, T. Li, T. Liu, C. Tong, and J. M. Yang, "Heavy standard model-like Higgs boson and a light stop from Yukawa-deflected gauge mediation," Physical Review D, vol. 86, no. 9, Article ID 095020, 2012.

[60] N. Craig, S. Knapen, D. Shih, and Y. Zhao, "A complete model of low-scale gauge mediation," Journal of High Energy Physics, vol. 154, 2013.

[61] M. B. Green and J. H. Schwarz, "Anomaly cancellations in supersymmetric $D=10$ gauge theory and superstring theory," Physics Letters B, vol. 149, no. 1-3, pp. 117-122, 1984. 
[62] M. B. Green and J. H. Schwarz, "The hexagon gauge anomaly in type 1 superstring theory," Nuclear Physics B, vol. 255, pp. 93-114, 1985.

[63] M. B. Green, J. H. Schwarz, and P. West, "Anomaly-free chiral theories in six dimensions," Nuclear Physics B, vol. 254, pp. 327348, 1985.

[64] H. K. Dreiner and M. Thormeier, "Supersymmetric FroggattNielsen models with baryon- and lepton-number violation," Physical Review D, vol. 69, no. 5, Article ID 053002, 2004.

[65] H. K. Dreiner, H. Murayama, and M. Thormeier, "Anomalous flavor $U(1)_{X}$ for everything," Nuclear Physics B, vol. 729, no. 1-2, pp. 278-316, 2005.

[66] H. K. Dreiner, C. Luhn, H. Murayama, and M. Thormeier, "Baryon triality and neutrino masses from an anomalous flavor U(1)," Nuclear Physics B, vol. 774, no. 1-3, pp. 127-167, 2007.

[67] I. Gogoladze, C.-A. Lee, T. Li, and Q. Shafi, "Fermion masses and mixings in grand unified theories with noncanonical $U(1)_{Y}$," Physical Review D, vol. 78, Article ID 015024, 2008.

[68] J. A. Evans, D. Shih, and A. Thalapillil, "Chiral flavor violation from extended gauge mediation," Journal of High Energy Physics, vol. 40, 2015.

[69] M. Abdullah, I. Galon, Y. Shadmi, and Y. Shirman, "Flavored gauge mediation, a heavy Higgs, and supersymmetric alignment," Journal of High Energy Physics, vol. 57, 2013.

[70] F. Staub, “Sarah,” https://arxiv.org/abs/0806.0538, 2008.

[71] F. Staub, "From superpotential to model files for FeynArts and CalcHep/CompHep," Computer Physics Communications, vol. 181, no. 6, pp. 1077-1086, 2010.

[72] F. Staub, "Automatic calculation of supersymmetric renormalization group equations and loop corrections," Computer Physics Communications, vol. 182, no. 3, pp. 808-833, 2011.

[73] F. Staub, "SARAH 3.2: Dirac gauginos, UFO output, and more," Computer Physics Communications, vol. 184, no. 7, pp. 17921809, 2013.

[74] F. Staub, "SARAH 4: A tool for (not only SUSY) model builders," Computer Physics Communications, vol. 185, no. 6, pp. 17731790, 2014.

[75] W. Porod, "SPheno, a program for calculating supersymmetric spectra, SUSY particle decays and SUSY particle production at $\mathrm{e}^{+} \mathrm{e}^{-}$colliders," Computer Physics Communications, vol. 153, pp. 275-315, 2003.

[76] W. Porod and F. Staub, "SPheno 3.1: extensions including flavour, CP-phases and models beyond the MSSM," Computer Physics Communications, vol. 183, no. 11, pp. 2458-2469, 2012.

[77] F. Staub, T. Ohl, W. Porod, and C. Speckner, "A tool box for implementing supersymmetric models," Computer Physics Communications, vol. 183, no. 10, pp. 2165-2206, 2012.

[78] V. Khachatryan, A. M. Sirunyan, A. Tumasya et al., "Observation of the rare $B_{s}^{0} \rightarrow \mu^{+} \mu^{-}$decay from the combined analysis of CMS and LHCb data," Nature, vol. 522, pp. 68-72, 2015.

[79] Y. Amhis, S. W. Banerjee, E. Ben-Haim et al., "Averages of $b$ hadron, $c$-hadron, and $\tau$-lepton properties as of summer 2014," https://arxiv.org/abs/1412.7515, 2014.

[80] ATLAS Collaboration, "Search for the Supersymmetric Partner of the Top Quark in the Jets+Emiss Final State at $\operatorname{sqrt}(\mathrm{s})=$ $13 \mathrm{TeV}$," ATLAS, Geneva, Switzerland, ATLAS-CONF-2016-077, 2016.

[81] CMS Collaboration, "Search for supersymmetry in the allhadronic final state using top quark tagging in pp collisions at sqrt(s) = 13 TeV," Tech. Rep., CMS-PAS-SUS-16-030, 2016.
[82] M. Aaboud, G. Aad, B. Abbott et al., "Search for bottom squark pair production in proton-proton collisions at $\sqrt{s}=13 \mathrm{TeV}$ with the ATLAS detector," The European Physical Journal C, vol. 76, no. 10, 547 pages, 2016.

[83] CMS Collaboration, "Search for new physics in the all-hadronic final state with the MT2 variable," Tech. Rep., Geneva, Switzerland, CMS-PAS-SUS-16-015, 2016.

[84] ATLAS Collaboration, "Further searches for squarks and gluinos in final states with jets and missing transverse momentum at $\sqrt{s}=13 \mathrm{TeV}$ with the ATLAS detector," Tech. Rep., ATLAS-CONF-2016-078, 2016.

[85] R. Barbieri and G. F. Giudice, "Upper bounds on supersymmetric particle masses," Nuclear Physics B, vol. 306, no. 1, pp. 63-76, 1988.

[86] J. Ellis, K. Enqvist, D. Nanopoulos, and F. Zwirner, “Observables in low-energy superstring models," Modern Physics Letters A, vol. 1, no. 1, pp. 57-69, 1986. 

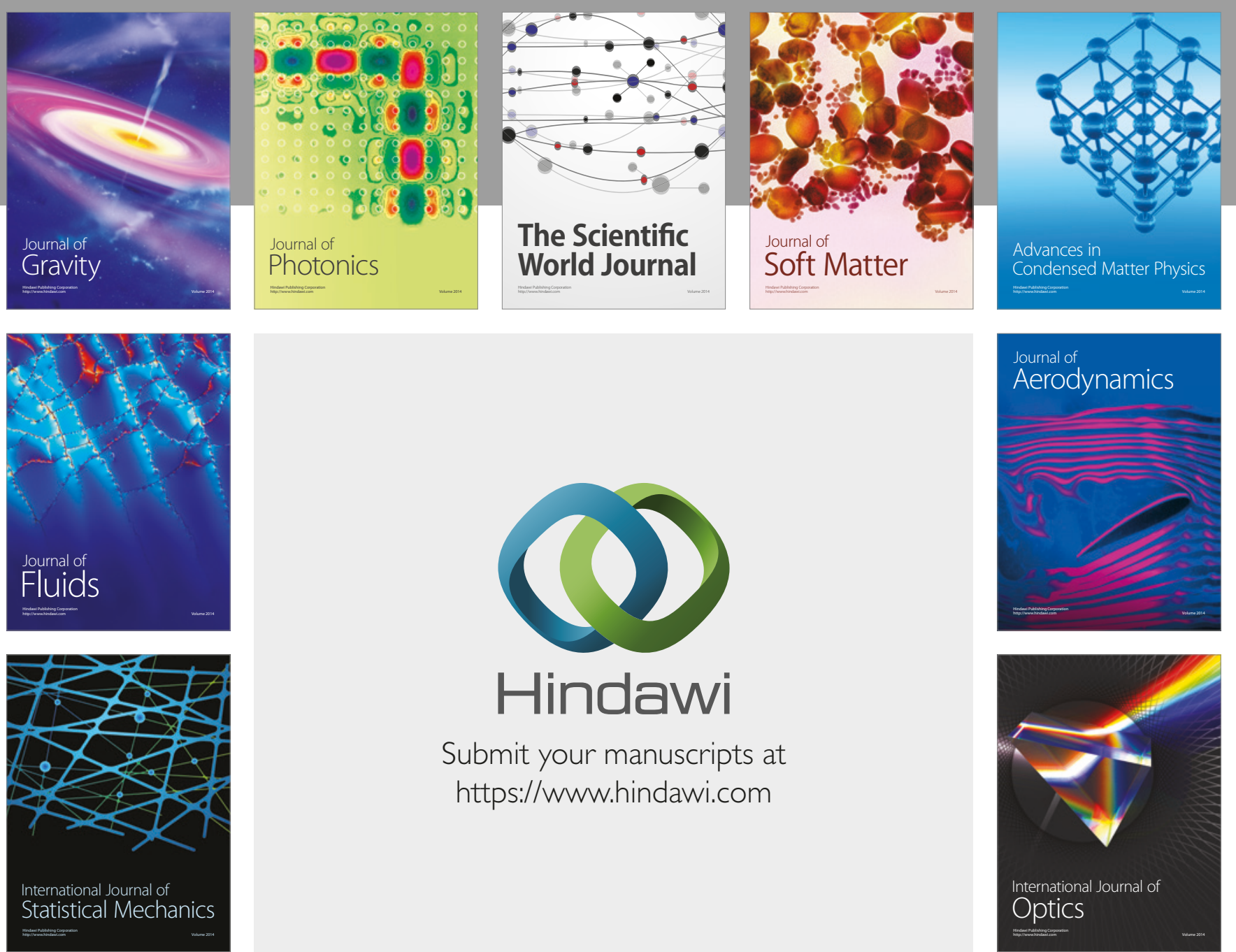

Submit your manuscripts at

https://www.hindawi.com
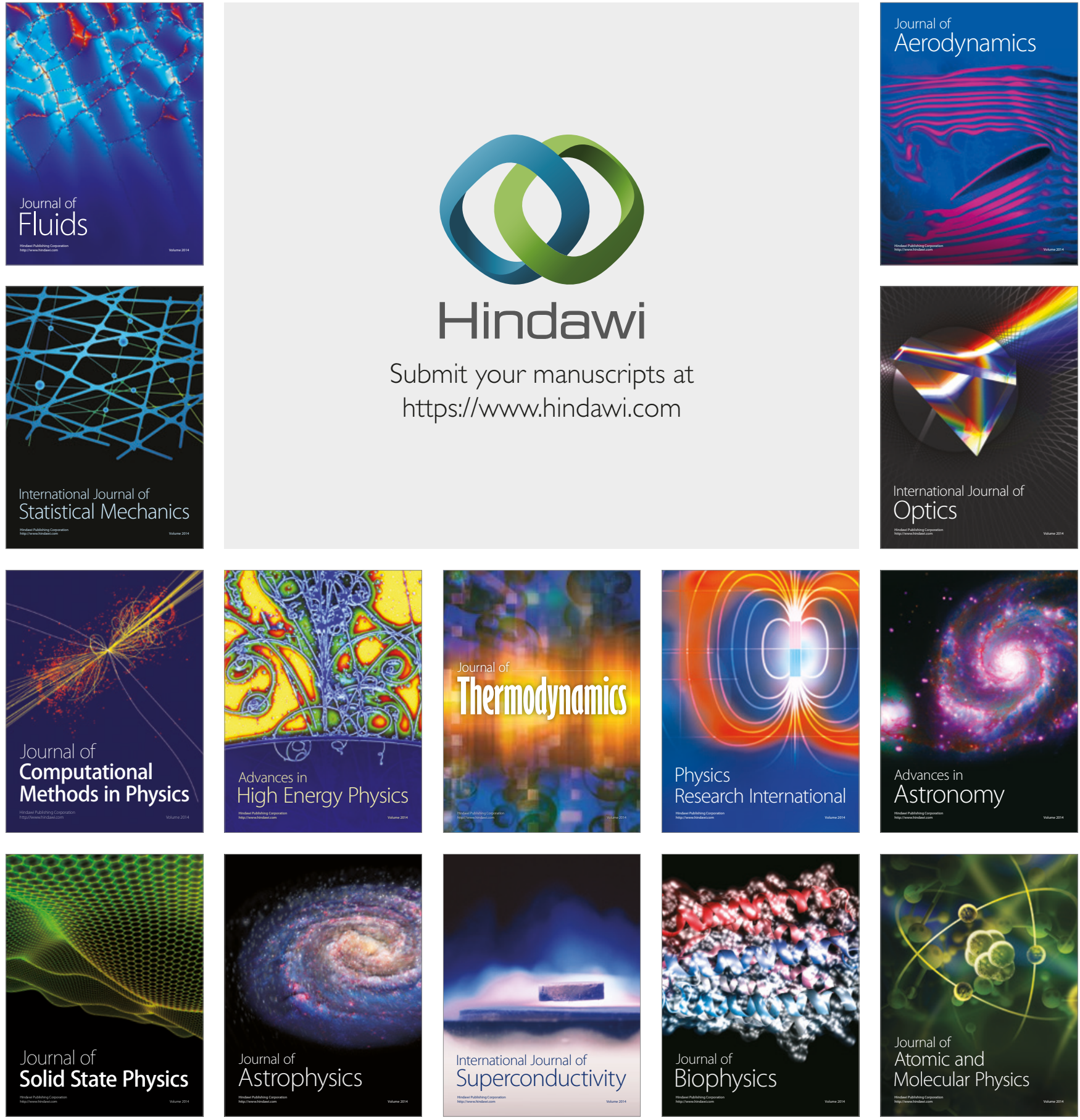